\title{
REFLECTION AND REFRACTION OF AN OBLIQUELY INCIDENT SHEAR WAVE AT A SOLID-NEMATIC INTERFACE
}

\author{
P. J. BARRATT and F. M. LESLIE \\ Department of Mathematics, Strathclyde University, Glasgow, Scotland
}

\begin{abstract}
Résumé. - Nous présentons un calcul détaillé de la réflexion et de la réfraction des ondes de cisaillement en incidence oblique sur un interface solide-nématique pour les trois géométries discutées par Martinoty et Candau [1]. Contrairement à l'analyse donnée par ces auteurs, lorsque l'inclinaison du détecteur en surface est parallèle au mouvement induit, nous trouvons dans le nématique trois modes plutôt que deux. Pour les deux autres géométries, nous trouvons simplement deux modes mais certains aspects de notre calcul sont nouveaux. Néanmoins, dans les trois cas, les résultats obtenus se réduisent à ceux donnés par Martinoty et Candau moyennant des approximations raisonnables.
\end{abstract}

\begin{abstract}
We present a detailed calculation of reffection and refraction of obliquely incident shear waves at a solid-nematic interface for the three surface alignments discussed by Martinoty and Candau [1]. In contrast with the analysis given by these authors, when the surface alignment is parallel to the induced motion, our solution for the perturbation in the nematic consists of three modes rather than two. While the solutions for the remaining two cases each consist of only two modes, certain aspects of our calculation are new. However, for all three cases, the results obtained reduce under reasonable approximations to those given by Martinoty and Candau.
\end{abstract}

1. Introduction. - In a paper presented at the Third International Liquid Crystal Conference in Berlin, Martinoty and Candau [1] describe a convenient method for the determination of certain viscosities of nematic liquid crystals. Their experiment involves the generation of a shear wave in a quartz crystal and the measurement of the wave reflected from a plane surface which supports a layer of the liquid crystal. Since the disturbance propagates a short distance into the adjacent liquid layer, information follows concerning the viscous properties of the nematic. These depend of course upon the particular alignment at the solid-nematic interface, and thus appropriate preparations of the surface lead to different viscosity measurements. A decided advantage of this method lies in its use of small samples of liquid crystal, with good control of alignment eliminating possible discrepancies due to the presence of defects (cf. remarks by de Gennes [2]). In their experiment, the shear wave is necessarily obliquely incident upon the interface, and the transverse displacement in the solid is parallel to this surface, which leads rather naturally to three viscosity measurements. In two the undisturbed uniform alignment is parallel to the interface, in one case parallel to the displacement and in the other perpendicular, and in the third it is normal to the surface. Initially, Martinoty and Candau obtained results for MBBA for the two surface alignments in the plane of the interface, but subsequently Kiry and Martinoty [3] have repeated the experiment for the nematic PCB for all three cases.

In their paper Martinoty and Candau present an analysis of the above experiment using the continuum theory proposed by Ericksen [4] and Leslie [5] for dynamic effects in nematic liquid crystals. They examine solutions corresponding to the three surface alignments just mentioned, but give details of the calculations only for that in which the initial orientation is parallel to the displacement, simply stating results for the remaining two. Considering first the rather special case of normal incidence for which the analysis is more straightforward, Martinoty and Candau obtain solutions of the linearised continuum equations which satisfy the appropriate interfacial conditions. In this case the refracted waves consist simply of a combination of the relevant fast and slow modes of the type described by the Orsay Group [6]. They go on to discuss the more relevant oblique incidence, and claim that this is also amenable to analysis in terms of two such modes. However, in this more general case with the initial alignment parallel to the displacement, it does not appear possible to satisfy all of the interfacial conditions in such an analysis, as Leslie [7] notes. Consequently, we present 
below a more detailed investigation of this particular case and show that it is necessary to include a third mode to obtain a satisfactory solution. On the other hand, for the remaining two surface alignments considered by Martinoty and Candau, there are appropriate solutions consisting of only two modes as they claim. Nonetheless, it seems desirable to give a brief account of our results for these two cases, since certain aspects of our calculation are new. However, it is important to emphasize that our results for oblique incidence reduce to those obtained originally by Martinoty and Candau when we use the low frequency approximation employed by these authors.

Since details of continuum theory and relevant physical properties of nematic liquid crystals are readily available in the books by de Gennes [2] and Chandrasekhar [8], and also in the reviews by Stephen and Straley [9], Ericksen [10] and Leslie [7], no further account appears necessary here. Therefore we proceed directly to the problem of interest.

2. Statement of problem. - For present purposes, we consider a plane interface between an elastic solid and a nematic liquid crystal, and regard both as unbounded, thereby ignoring the influence of all other boundaries. In the solid, a plane shear wave of small amplitude is incident upon the interface at an angle $\varphi$ with the normal, producing a second reflected wave. Our object here is to determine the nature of the consequent disturbance in the nematic and its influence upon the reflected wave. The displacement in the solid must satisfy the equations of classical, linear, isotropic elasticity, while the perturbation of the liquid crystal is governed by the linearised version of the continuum equations for nematics. At the interface, displacement and surface traction are continuous, and we assume that the anisotropic axis is strongly anchored. A natural choice of Cartesian axes for this problem takes the plane $z=0$ coincident with the interface, with the positive $z$-axis into the nematic and the $x$-axis parallel to the displacement in the solid.

The relevant solution for the displacement vector $\mathbf{u}$ in the solid corresponding to the incident and reflected waves is therefore

$$
\begin{aligned}
& u_{x}=A \exp i[\omega t-k(y \sin \varphi+z \cos \varphi)]+ \\
&+B \exp i[\omega t-k(y \sin \varphi-z \cos \varphi)], \\
& u_{y}=u_{z}=0
\end{aligned}
$$

where the small amplitudes $A$ and $B$, the frequency $\omega$ and the wave number $k$ are all constants, and $t$ denotes time. The first term of course represents the incident wave, and the second the reflected wave. The frequency and wave number are not independent but are related through

$$
\omega / k=c=(\mu / \rho)^{1 / 2},
$$

$\mu$ being the rigidity modulus, $\rho$ density and $c$ the wave speed.
In view of the above, the disturbance in the nematic is subject to the following boundary conditions. At the interface, the velocity vector $\mathbf{v}$ must satisfy

$$
\begin{gathered}
v_{x}=i \omega(A+B) \exp i(\omega t-k y \sin \varphi), \\
v_{y}=v_{z}=0,
\end{gathered}
$$

and the surface traction $t$ exerted by the nematic upon the solid takes the form

$$
\begin{gathered}
t_{x}=i \mu k \cos \varphi(B-A) \exp i(\omega t-k y \sin \varphi), \\
t_{y}=t_{z}=0 .
\end{gathered}
$$

Also, given the strong anchoring assumption, any perturbation of the anisotropic axis necessarily vanishes at the interface.

The choice of solution corresponding to the disturbance in the nematic of course depends upon the particular surface alignment under consideration. In all cases, however, we examine velocity perturbations of the form

$v_{x}=a \exp i[\omega t-k(y \sin \varphi+q z)], \quad v_{y}=v_{z}=0$,

where $a$ and $q$ are constants to be determined, the former small. When the surface alignment is parallel to the displacement vector, it proves necessary to consider solutions in which the anisotropic axis $\mathbf{n}$ is given by

$$
\begin{gathered}
n_{x}=1, \quad n_{y}=m \exp i[\omega t-k(y \sin \varphi+q z)], \\
n_{z}=n \exp i[\omega t-k(y \sin \varphi+q z)],
\end{gathered}
$$

with $m$ and $n$ small constants. On the other hand, when the surface alignment is normal to the displacement and either parallel or perpendicular to the interface, it suffices to discuss disturbances to this axis of the type

$$
\begin{gathered}
n_{x}=n \exp i[\omega t-k(y \sin \varphi+q z)], \\
n_{y}=1, \quad n_{z}=0,
\end{gathered}
$$

or

$$
\begin{gathered}
n_{x}=n \exp i[\omega t-k(y \sin \varphi+q z)], \\
n_{y}=0, \quad n_{z}=1,
\end{gathered}
$$

respectively, where $\boldsymbol{n}$ is again a small constant.

Some understanding of the above choices for the anisotropic axis stems from the following considerations. Given the spatial dependence of the induced motion, an initial alignment parallel to the flow experiences torques leading to small perturbations in both the $y$ and $z$ directions. However, the remaining two cases are somewhat exceptional in that each is insensitive to one component of the shear. For example, alignment parallel to the $y$-axis is unaffected by the $z$ dependence of the induced motion, and therefore to first order only the perturbation in 
the $x$ direction couples with the shear. Similarly, alignment normal to the interface experiences no torque on account of the $y$ dependence of the flow, and again only a perturbation in the $x$ direction occurs.

The above expressions must of course satisfy the appropriate continuum equations for nematics. Here, unlike Martinoty and Candau, we do not set the Frank coefficients equal since the analysis proceeds straightforwardly without this simplification. On the other hand, we do conform with common practice and adopt the Parodi relation [11], although this is not essential for our results.

3. Solution with three modes. - For the case of surface alignment parallel to the induced flow, the expressions (5) and (6) are solutions of the relevant equations provided that

$$
\begin{gathered}
\left(K_{2} q^{2}+K_{1} \sin ^{2} \varphi+i \gamma_{1} \xi\right) k m+\left(K_{1}-K_{2}\right) k q \sin \varphi n \\
-i \alpha_{3} \sin \varphi a=0, \\
\left(K_{1}-K_{2}\right) k q \sin \varphi m+\left(K_{1} q^{2}+K_{2} \sin ^{2} \varphi+i \gamma_{1} \xi\right) k n \\
-i \alpha_{3} q a=0, \\
{\left[\eta_{b}\left(q^{2}+\sin ^{2} \varphi\right)+i \rho \xi\right] k a-\alpha_{3} \omega \sin \varphi m}
\end{gathered}
$$$$
-\alpha_{3} \omega q n=0
$$

where

$$
2 \eta_{b}=\alpha_{4}+\alpha_{3}+\alpha_{6}, \quad \xi=\omega / k^{2}
$$

In these equations, the $K$ 's denote the Frank coefficients in the free energy and $\gamma_{1}$ and the $\alpha$ 's are viscosity coefficients. With the additional notation

$$
\tilde{\eta}_{b}=\eta_{b}-\alpha_{3}^{2} / \gamma_{1}, \quad \mu_{b}=\rho K_{1} / \gamma_{1} \tilde{\eta}_{b},
$$

the determinantal condition for non-trivial solutions of equations (9) reduces to

$$
\begin{aligned}
& {\left[K_{2}\left(q^{2}+\sin ^{2} \varphi\right)+i \gamma_{1} \xi\right]\left[\eta_{b} K_{1}\left(q^{2}+\sin ^{2} \varphi\right)^{2}+\right.} \\
& \left.\quad+i \gamma_{1} \xi \widetilde{\eta}_{b}\left(1+\mu_{b}\right)\left(q^{2}+\sin ^{2} \varphi\right)-\rho \gamma_{1} \xi^{2}\right]=0
\end{aligned}
$$

which is cubic in the square of the unknown parameter $q$. Hence there are in general three possible values for this quantity yielding solutions which remain finite far from the interface.

By superposition, therefore, one obtains

$$
\left.\begin{array}{l}
v_{x}=\sum_{r=1}^{3} a_{r} \exp i\left[\omega t-k\left(y \sin \varphi+q_{r} z\right)\right], \\
n_{y}=\sum_{r=1}^{3} m_{r} \exp i\left[\omega t-k\left(y \sin \varphi+q_{r} z\right)\right], \\
n_{z}=\sum_{r=1}^{3} n_{r} \exp i\left[\omega t-k\left(y \sin \varphi+q_{r} z\right)\right] .
\end{array}\right\}
$$

When $q$ is equal to $q_{1}$ or $q_{2}$, the first two members of equations (9) quickly yield

$$
q_{r} m_{r}=\sin \varphi n_{r}, \quad r=1,2,
$$

and thus it follows from the third that

$$
\begin{aligned}
& {\left[\eta_{b}\left(q_{r}^{2}+\sin ^{2} \varphi\right)+i \rho \xi\right] k q_{r} a_{r}=} \\
& =\alpha_{3} \omega\left(q_{r}^{2}+\sin ^{2} \varphi\right) n_{r}, \quad r=1,2 .
\end{aligned}
$$

When $q$ takes the value $q_{3}$, however, the equations (9) reduce to

$$
\left.\begin{array}{r}
k\left(K_{1}-K_{2}\right)\left(\sin \varphi m_{3}+q_{3} n_{3}\right)-i \alpha_{3} a_{3}=0 \\
\alpha_{3} \omega K_{2}\left(\sin \varphi m_{3}+q_{3} n_{3}\right)+ \\
+i \xi k\left(\eta_{b} \gamma_{1}-\rho K_{2}\right) a_{3}=0
\end{array}\right\}
$$

from which one readily deduces that

$$
\sin \varphi m_{3}=-q_{3} n_{3}, \quad a_{3}=0,
$$

unless

$$
\tilde{\eta}_{b}-K_{1} \eta_{b} / K_{2}+\rho\left(K_{1}-K_{2}\right) / \gamma_{1}=0 .
$$

For the moment, we assume that the material parameters do not satisfy this last condition, and therefore that the coefficients in the third mode are subject to the restrictions (17).

In view of the strong anchoring assumption at the interface, one must have

$$
m_{1}+m_{2}+m_{3}=0, \quad n_{1}+n_{2}+n_{3}=0,
$$

and these together with equations (14) and (17) combine to yield

$$
\left(q_{1} q_{3}+\sin ^{2} \varphi\right) q_{2} n_{1}+\left(q_{2} q_{3}+\sin ^{2} \varphi\right) q_{1} n_{2}=0 .
$$

With the relations (15), this last equation gives the ratio of the coefficients $a_{1}$ and $a_{2}$. The interfacial conditions (3) and (4) lead to the further equations

$$
\left.\begin{array}{c}
i \omega(A+B)=a_{1}+a_{2}, \\
\mu \cos \varphi(A-B)=\eta_{b}\left(q_{1} a_{1}+q_{2} a_{2}\right),
\end{array}\right\}
$$

and hence

$(A-B) /(A+B)=i \omega \eta_{b}\left(q_{1} a_{1}+q_{2} a_{2}\right) / \mu \cos \varphi\left(a_{1}+a_{2}\right)$.

Since the ratio of $a_{2}$ to $a_{1}$ is known, this last equation determines the amplitude of the reflected wave in terms of that of the incident wave and therefore completes our solution.

As for example de Gennes [2] or Leslie [7] discuss, the material parameters $K_{1}, K_{2}, \eta_{b}, \widetilde{\eta}_{b}$ and $\gamma_{1}$ are all positive. Moreover, since the Frank constants are in general very small in magnitude compared with the viscosity coefficients, we assume in what follows that

$$
\rho K / \gamma_{1} \eta \ll 1, \quad K \ll \gamma_{1} \omega / k^{2},
$$

where $K$ denotes either of the elastic coefficients, and $\eta$ represents $\eta_{b}$ or $\tilde{\eta}_{b}$. Our first assumption is 
identical to that made by the Orsay Group [6]. With these assumptions, the roots of equation (12) are to a good approximation given by

$$
\begin{gathered}
q_{1}^{2}=-\sin ^{2} \varphi-i \rho \omega / \tilde{\eta}_{b} k^{2}, \\
q_{2}^{2}=-i \gamma_{1} \tilde{\eta}_{b} \omega / \eta_{b} K_{1} k^{2}, \quad q_{3}^{2}=-i \gamma_{1} \omega / K_{2} k^{2},
\end{gathered}
$$

where the $\sin ^{2} \varphi$ term is omitted in the last two expressions since the other term dominates. Also, in this approximation, the equations (15), (20) and (24) yield

$$
\left(\widetilde{\eta}_{b}-\eta_{b}\right) q_{1} a_{1}=\eta_{b} q_{2} a_{2},
$$

and similarly the expression (22) reduces to

$$
(A-B) /(A+B)=i \omega \tilde{\eta}_{b} q_{1} / \mu \cos \varphi,
$$

which differs from the result obtained by Martinoty and Candau only through the retention of the $\sin ^{2} \varphi$ term in the first of equations (24).

Finally, we note that with the approximations (23), the condition (18) takes the simpler form

$$
K_{1} / K_{2}=\tilde{\eta}_{b} / \eta_{b}
$$

In view of earlier remarks concerning the material parameters, $\widetilde{\eta}_{b}$ is smaller than $\eta_{b}$ and thus the right hand side of the above is less than unity. Since the splay elastic constant is invariably larger than the twist constant, our adoption of the solution (17) is justified, except possibly in the neighbourhood of a smectic transition.

4. Solutions with two modes. - When the surface alignment is parallel to the interface but perpendicular to the induced motion, the expressions (5) and (7) are relevant solutions provided that

$$
\begin{aligned}
\left(K_{2} q^{2}+K_{3} \sin ^{2} \varphi+i \gamma_{1} \xi\right) k n & -i \alpha_{2} \sin \varphi a=0, \\
\left(\eta_{a} q^{2}+\eta_{c} \sin ^{2} \varphi+i \rho \xi\right) & k a \\
- & -\alpha_{2} \omega \sin \varphi n=0,
\end{aligned}
$$

where

$$
2 \eta_{a}=\alpha_{4}, \quad 2 \eta_{c}=\alpha_{4}+\alpha_{5}-\alpha_{2} .
$$

In this case, with the notation

$$
\left.\begin{array}{c}
\tilde{\eta}_{c}=\eta_{c}-\alpha_{2}^{2} / \gamma_{1}, \\
\mu_{a}=\rho K_{2} / \gamma_{1} \eta_{a}, \quad \mu_{c}=\rho K_{3} / \gamma_{1} \tilde{\eta}_{c},
\end{array}\right\}
$$

the associated determinantal condition reduces to

$\eta_{a} K_{2} q^{4}+\left[\left(\eta_{a} K_{3}+\eta_{c} K_{2}\right) \sin ^{2} \varphi+i \gamma_{1} \xi \eta_{a}\left(1+\mu_{a}\right)\right] q^{2}+$

$+K_{3} \eta_{c} \sin ^{4} \varphi+i \gamma_{1} \xi \tilde{\eta}_{c}\left(1+\mu_{c}\right) \sin ^{2} \varphi-\rho \gamma_{1} \xi^{2}=0$,

which is quadratic in the square of $q$ leading to simply two modes. Here, therefore, the solutions are

$$
\left.\begin{array}{l}
v_{x}=\sum_{r=1}^{2} a_{r} \exp i\left[\omega t-k\left(y \sin \varphi+q_{r} z\right)\right], \\
n_{x}=\sum_{r=1}^{2} n_{r} \exp i\left[\omega t-k\left(y \sin \varphi+q_{r} z\right)\right],
\end{array}\right\}
$$

and it follows from equations (28) that

$$
\begin{aligned}
& \left(\eta_{a} q_{r}^{2}+\eta_{c} \sin ^{2} \varphi+i \rho \xi\right) k a_{r}= \\
& =\alpha_{2} \omega \sin \varphi n_{r}, \quad r=1,2 .
\end{aligned}
$$

Also, the interfacial condition upon the anisotropic axis yields

$$
n_{1}+n_{2}=0,
$$

which with the previous equations determines the ratio of the coefficients in the velocity component. Finally, one obtains the relationships (21) with $\eta_{a}$ replacing $\eta_{b}$ from the remaining interfacial conditions.

With the assumptions (23), the roots of the above quadratic are given to a good approximation by

$q_{1}^{2}=-i \rho \omega / \eta_{a} k^{2}-\tilde{\eta}_{c} \sin ^{2} \varphi / \eta_{a}, \quad q_{2}^{2}=-i \gamma_{1} \omega / K_{2} k^{2}$,

and the equations (33) and (34) combine to yield

$$
i \gamma_{1} \eta_{a} \omega a_{2}=K_{2}\left(\eta_{c}-\tilde{\eta}_{c}\right) k^{2} \sin ^{2} \varphi a_{1}
$$

Here, therefore, one finds in this approximation that

$$
q_{1} / q_{2} \sim \mu_{a}^{1 / 2}, \quad a_{2} / a_{1} \sim K_{2} k^{2} / \gamma_{1} \omega,
$$

and finally that

$$
(A-B) /(A+B)=i \omega \eta_{a} q_{1} / \mu \cos \varphi,
$$

from which the coefficient $B$ follows in terms of $A$ and material parameters.

For the remaining case of surface alignment normal to the interface, the equations (5) and (8) are appropriate solutions if

$$
\left.\begin{array}{cc}
\left(K_{3} q^{2}+K_{2} \sin ^{2} \varphi-i \gamma_{1} \xi\right) k n & -i \alpha_{2} q a=0, \\
\left(\eta_{c} q^{2}+\eta_{a} \sin ^{2} \varphi+i \rho \xi\right) k a & \\
& -\alpha_{2} \omega q n=0,
\end{array}\right\}
$$

from which one readily obtains the equation

$$
\begin{gathered}
K_{3} \eta_{c} q^{4}+\left[\left(\eta_{c} K_{2}+\eta_{a} K_{3}\right) \sin ^{2} \varphi+i \gamma_{1} \xi \tilde{\eta}_{c}\left(1+\mu_{c}\right)\right] q^{2}+ \\
+\left(\eta_{a} \sin ^{2} \varphi+i \rho \xi\right)\left(K_{2} \sin ^{2} \varphi+i \gamma_{1} \xi\right)=0
\end{gathered}
$$

For this surface alignment, therefore, our solution is also of the form (32) with the equations (39) yielding

$\left(\eta_{c} q_{r}^{2}+\eta_{a} \sin ^{2} \varphi+i \rho \xi\right) k a_{r}=\alpha_{2} \omega q_{r} n_{r}, \quad r=1,2$, 
and as before (34) and (21) with $\eta_{c}$ replacing $\eta_{b}$ follow from the interfacial conditions.

The assumptions (23) in this case lead to the approximate roots

$$
\begin{gathered}
q_{1}^{2}=-i \rho \omega / \tilde{\eta}_{c} k^{2}-\eta_{a} \sin ^{2} \varphi / \tilde{\eta}_{c} \\
q_{2}^{2}=-i \gamma_{1} \tilde{\eta}_{c} \omega / K_{3} \eta_{c} k^{2}
\end{gathered}
$$

and equations (34) and (41) combine to give

$$
\begin{aligned}
K_{3} q_{2}\left(k^{2} \eta_{a} \sin ^{2} \varphi+i \rho \omega\right)\left(\tilde{\eta}_{c}\right. & \left.-\eta_{c}\right) a_{1}= \\
& =i \gamma_{1} \tilde{\eta}_{c}^{2} q_{1} \omega a_{2} .
\end{aligned}
$$

Consequently, to a good approximation, one finds that

$$
q_{2} a_{2} / q_{1} a_{1}=\left(\tilde{\eta}_{c}-\eta_{c}\right) / \eta_{c},
$$

and lastly that

$$
(A-B) /(A+B)=i \omega \tilde{\eta}_{c} q_{1} / \mu \cos \varphi,
$$

which again determines $B$.
5. Conclusions. - Given the merits of the Martinoty-Candau experiment as a means of evaluating nematic viscosities, it is important that the associated theory has a sound basis. The preceding analysis presents detailed solutions for the case of oblique incidence for the three surface alignments employed in these experiments. When this is parallel to the induced motion, it proves necessary to introduce a third mode over and above the two analogous to those which occur in Martinoty and Candau's analysis of normal incidence. However, while this is essential in order to satisfy all of the interfacial conditions, the net effect is not very significant.

Finally, for frequencies $\omega$ satisfying

$$
c^{2} / \omega \gg \eta / \rho,
$$

where $\eta$ denotes $\eta_{a}, \tilde{\eta}_{b}$ or $\tilde{\eta}_{c}$, our principal results (26), (38) and (45) reduce to those found by Martinoty and Candau for oblique incidence. Therefore, under conditions pertaining to their experiments our final conclusions coincide.

\section{References}

[1] Martinoty, P. and Candau, S., Mol. Cryst. Liq. Cryst. 14 (1971) 243.

[2] De Gennes, P. G., The Physics of Liquid Crystals (Clarendon Press, Oxford) 1974.

[3] KIRY, F. and Martinoty, P., J. .Physique 38 (1977) 153.

[4] Ericksen, J. L., Trans. Soc. Rheol. 5 (1961) 23.

[5] Leslie, F. M., Arch. Rat. Mech. Anal. 28 (1968) 265.

[6] Orsay Liquid Crystal Group, J. Chem. Phys. 51 (1969) 816.
[7] LesLie, F. M., Adv. Liquid Crystals (to appear).

[8] Chandrasekhar, S., Liquid Crystals (University Press, Cambridge) 1977.

[9] Stephen, M. J. and Straley, J. P., Rev. Mod. Phys. 46 (1974) 617.

[10] ERICKsen, J. L., Adv. Liquid Crystals 2 (1976) 233.

[11] Parodi, O., J. Physique 31 (1970) 581. 